\title{
Safety and Efficacy of Neoadjuvant DOF [Docetaxel, Oxaliplatin, 5-Fluorouracil] Chemotherapy Regimen in Patients with Locally Advanced Gastric and Gastro-Esophageal Junction Cancers: A Single Center Experience from India
}

\author{
Vishal Kulkarni, Satheesh Chiradoni Thungappa, Shekhar Patil, Vinu Sarathy*, \\ Kiran Pura Krishnamurthy, Rajesh Kumar, Radheshyam Naik
}

Department of Medical Oncology, Health Care Global Enterprises Ltd., Bangalore, India

Email: *sarathy.vinu.88@gmail.com

How to cite this paper: Kulkarni, V., Thungappa, S.C., Patil, S., Sarathy, V., Krishnamurthy, K.P., Kumar, R. and Naik, R. (2020) Safety and Efficacy of Neoadjuvant DOF [Docetaxel, Oxaliplatin, 5-Fluorouracil] Chemotherapy Regimen in Patients with Locally Advanced Gastric and Gastro-Esophageal Junction Cancers: A Single Center Experience from India. Journal of Cancer Therapy, 11, 237-250.

https://doi.org/10.4236/jct.2020.115020

Received: March 3, 2020

Accepted: April 25, 2020

Published: April 28, 2020

Copyright ( 2020 by author(s) and Scientific Research Publishing Inc. This work is licensed under the Creative Commons Attribution International License (CC BY 4.0).

http://creativecommons.org/licenses/by/4.0/ (c) (i) Open Access

\section{Abstract}

Background: The role of chemotherapy in Gastric Cancer is constantly evolving with various neoadjuvant and adjuvant strategies. Several chemotherapeutic agents are used in the treatment of locally advanced gastric cancer (LAGC) namely Platinum based compounds (Cisplatin, Oxaliplatin), Fluoropyrimidines like 5-Flurouracil [(5-FU), Capecitabine)], Taxanes (Docetaxel) and Anthracyclines (Epirubicin). Various doublet and triplet combination chemotherapy regimens have been used for neo-adjuvant chemotherapy (NACT) in LAGCs. In this study we evaluated the safety and efficacy of docetaxel based triplet regimen DOF [Docetaxel, Oxaliplatin, 5-Fluorouracil] in LAGC. Material and methods: 50 Newly diagnosed patients of Locally Advanced Gastric Cancer (stage II or III) deemed fit to receive chemotherapy were included in our study. After 3 cycles of neoadjuvant chemotherapy, patients were assessed based on radiological and pathological response. Results: 50 Patients were included in our study of which majority were male (32), median age at presentation was 55 years and 24 patients presented with a history of gastrointestinal reflux disease (GERD). The most common hematological toxicities observed in our study were anemia (61.2\%), neutropenia (42.6\%, febrile neutropenia constituted 6\%) and thrombocytopenia (13.2\%). The most common gastro-intestinal [GI] toxicities observed in our study included nausea $(69.2 \%)$, vomiting $(31.2 \%)$, diarrhea (34\%), oral mucositis (14\%) and 
constipation (6.6\%). We found that safety profile of DOF regimen was favorable with majority of patients tolerating the regimen well. The Overall Response Rate (68\%), Disease Control Rate (96\%) and Resectability Rate (80\%) were higher compared to western studies. Pathological CR (17.5\%), $y p N_{0}$ disease status (42.5\%) and nodal down staging (52\%), all showed positive correlations with survival outcomes. Conclusion: DOF regimen is an effective and feasible option for neoadjuvant treatment of LAGC in an Indian population.

\section{Keywords}

Locally Advanced Gastric Cancer (LAGC), Neoadjuvant Chemotherapy, DOF (Docetaxel, Oxaliplatin, 5-Fluorouracil), Safety, Toxicity

\section{Introduction}

Gastric cancer (GC) is the fifth most common malignancy and third leading cause of cancer related deaths worldwide. Highest rates of incidence are seen in Eastern Asia, Eastern Europe and South America. In Asia the highest incidences are seen in China, Japan and Korea [1] [2]. In India, the north eastern region of Mizoram has the highest rates followed by Chennai, Bangalore and Hyderabad. The Incidence is lesser in Northern Indian as compared to the South [3]. Gastric cancer demonstrates familial aggregation in approximately $10 \%$ of cases and an inherited genetic predisposition in a small proportion (approximately $1 \%-3 \%$ ) [2] [3] [4]. Locally advanced gastric cancer (LAGC) includes AJCC/UICC stage II and stage III patients. About two-thirds of patients are diagnosed with LAGC at diagnosis which leads to significant morbidity and mortality [5] [6].

The role of chemotherapy in gastric cancer (GC) is constantly evolving to improve outcomes and reduce toxicity. Currently several acceptable chemotherapy approaches are available for management of LAGCs namely adjuvant (postoperative chemotherapy), peri-operative (pre and post-operative) and the most recent being neo-adjuvant chemotherapy (pre-operative chemotherapy). Several chemotherapeutic agents are used in treatment of GC namely platinum based compounds (Cisplatin, Oxaliplatin), fluoropyrimidines like 5-Flurouracil (5-FU) and Capecitabine, taxanes (Docetaxel) and anthracyclines (Epirubicin). Triplet regimens are more effective than doublet regimens for LAGCs. Some of the most commonly used triplet regimens are Epirubicin and Docetaxel based regimens The landmark FLOT-4 trial, a multi-centric randomized phase- 3 trial conducted by Al Batran et al. compared docetaxel-based triplet FLOT (modified DOF) with Anthracycline-based triplet Epirubicin, Cisplatin, and 5-Fluorouracil or Capecitabine (ECF/ECX) as perioperative treatment for patients with resectable gastric or GEJ cancers. Perioperative chemotherapy with Docetaxel, Oxaliplatin, and 5-Fluorouracil (FLOT) significantly improved Progression-Free Survival (PFS) and Overall Survival (OS) among patients with resectable gastric cancers compared with ECF/ECX. Of 716 patients enrolled, 360 patients received ECF/ECX 
and 356 patients received FLOT. After a median follow up of 43 months, median OS was 35 months with ECF/ECX and 50 months with FLOT (hazard ratio, 0.77; $P=0.012)$. Perioperative complications were similar across the 2 arms: $50 \%$ with ECF/ECX and 51\% with FLOT. More cases of grade 3/4 nausea and vomiting were seen with ECF/ECX and more cases of grade 3/4 neutropenia were seen with FLOT [7].

Despite data on improved overall survival and better compliance, DOF as NACT remains an experimental approach in India owing to limited number of studies conducted in India. As most DOF based trials have shown positive outcomes in the West and China, these results cannot be generalized due to population heterogeneity, difference in tumor characteristics and guidelines [8] [9]. Hence further evaluation of efficacy and safety of Neoadjuvant DOF regimen in Indian population would aid in optimizing treatment guidelines for LAGC. The present study was done to assess safety and efficacy of DOF regimen as Neoadjuvant chemotherapy in Locally Advanced Gastric Cancers.

\section{Materials and Methods}

This was a prospective, observational study. 50 newly diagnosed patients of Locally Advanced Gastric Cancer (stage II or III) assessed in our hospital from September 2016 to September 2017 deemed fit to receive chemotherapy were included in the study after taking prior informed consent. Fitness was determined by ECOG (Eastern Co-operative Oncology Group) Performance Status. Only those patients with ECOG $\leq 2$ were enrolled in the study. Patients with an ECOG $>2$, early or metastatic disease and those who underwent upfront surgery were excluded. A baseline PET-CT scans/CT scan was done for all the patients. Clinical Staging was recorded at baseline based on radiology reports.

\subsection{Statistical Methods}

The information collected was recorded on a master chart. The Statistical analysis was performed on a computer using SPSS 23.0. In Descriptive statistics, the continuous variables were expressed as Mean and Standard deviation for normally distributed data and median and range for skewed data. Categorical variables were expressed as frequency and percentage. Based on the normality of data, Chi-square was used to find association between the categorical variables and Pearson Co-relation Test was used to find the relationship between two variables. Independent Student $\mathrm{t}$ Test was used to find the difference between two groups. One way ANOVA test was used to find the difference between multiple groups. Results were graphically represented where deemed necessary. $\mathrm{P}<0.05$ was considered as statistically significant.

\subsection{Treatment Protocol}

DOF Regiment: D-Docetaxel $60 \mathrm{mg} / \mathrm{m}^{2}$ [D1] IV infusion over 2 hours, O-Oxaliplatin $100 \mathrm{mg} / \mathrm{m}^{2}$ [D1] IV infusion over 2 hours, $\mathrm{F}-5 \mathrm{FU} 750 \mathrm{mg} / \mathrm{m}^{2}$ [D1] [D2, 
D3] IV infusion over 6 hours. Each patient received 3 cycles of chemotherapy with DOF regimen as mentioned above. In each cycle patients were administered growth factor [Inj Pegylated GCSF $6 \mathrm{mg}$ SC], 24 hours after the end of chemotherapy. Detailed history, physical examination and investigations were done before each cycle. Laboratory results were recorded and the various hematological side effects of chemotherapeutic agents were analyzed. Patients were administered chemotherapy only if considered fit by the treating Medical Oncologist.

\subsection{Evaluation of the Safety Profile}

The grading used was according to CTCAE 4.03 criteria. Adverse effects were assessed before each cycle of chemotherapy and in the event of any patient reported issue.

\subsection{Dose Modifications}

Dose modifications were carried out based on the presence of any grade 3 or grade 4 side effects. $20 \%$ dose reductions were made for any grade $3 /$ grade 4 toxicity. If the patient required more than two dose reductions, treatment was discontinued.

\subsection{Evaluation of Efficacy}

At the end of three cycles the patients underwent a PET CT scan/CT scan to assess the response. The response assessment was based on the RECIST 1.1 criteria.

\section{Results}

\subsection{Patient Characteristics}

Fifty patients of newly diagnosed locally advanced gastric cancer were administered DOF regimen. The mean age was $55 \pm 7.97$ years. The number of male patients $(n=32)[64 \%]$ was higher compared to females $(n=18,36 \%)$. Number of patients with history of GERD (Gastro-Esophageal Reflux Disease) ( $\mathrm{n}=24,48 \%$ ) was almost similar to those without GERD ( $n=26,52 \%)$.

\subsection{Tumor Characteristics}

Of the 50 patients, the primary site was gastric (body, antrum and pylorus) in 41 patients (82\%), 6 patients (12\%) with involvement of both gastric and GastroEsophageal Junction [GEJ] and 3 (6\%) with purely GEJ tumor. 17 (34\%) patients presented with stage IIIA disease, 12 (24\%) IIIB, 11 (22\%) stage IIB, 7 (14\%) stage IIIC and the remaining $3(6 \%)$ with stage IIA disease (Table 1).

\subsection{Safety Profile Parameters}

\subsubsection{Hematological, Gastrointestinal and Other Toxicity Profiles}

Hematological toxicities are summarized in Table 2. Post cycle 1, the most 
Table 1. Distribution of tumor characteristics at baseline $(n=50)$.

\begin{tabular}{|c|c|c|c|}
\hline \multicolumn{4}{|c|}{ Distribution of tumor characteristics at baseline $(n=50)$} \\
\hline & & $\mathbf{n}$ & $\%$ \\
\hline & Gastric & 41 & $82 \%$ \\
\hline \multirow[t]{4}{*}{ Primary Site } & $\mathrm{GEJ}^{*}$ & 3 & $6 \%$ \\
\hline & Gastric + GEJ & 6 & $12 \%$ \\
\hline & IIA & 3 & $6 \%$ \\
\hline & IIB & 11 & $22 \%$ \\
\hline \multirow[t]{3}{*}{ Stage $\mathrm{e}^{\#}$} & IIIA & 17 & $34 \%$ \\
\hline & IIIB & 12 & $24 \%$ \\
\hline & IIIC & 7 & $14 \%$ \\
\hline
\end{tabular}

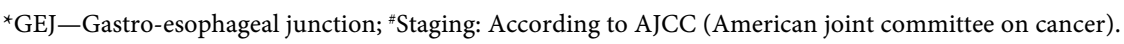

Table 2. Chemotherapy associated hematological toxicities observed in our study group.

\begin{tabular}{|c|c|c|c|c|c|c|c|c|c|}
\hline \multirow{3}{*}{ Chemotherapy } & \multirow{3}{*}{$\begin{array}{c}\text { Toxicity } \\
\text { Grade }\end{array}$} & \multicolumn{8}{|c|}{ Hematological Toxicities } \\
\hline & & \multicolumn{2}{|c|}{ Neutropenia } & \multicolumn{2}{|c|}{ Anemia } & \multicolumn{2}{|c|}{ Thrombocytopenia } & \multicolumn{2}{|c|}{$\begin{array}{c}\text { Febrile } \\
\text { neutropenia }\end{array}$} \\
\hline & & $\mathrm{n}$ & $\%$ & $\mathbf{n}$ & $\%$ & $\mathbf{n}$ & $\%$ & $\mathbf{n}$ & $\%$ \\
\hline \multirow{5}{*}{ Cycle 1} & Grade 1 & 3 & $6 \%$ & 27 & $54 \%$ & 2 & $4 \%$ & 0 & $0 \%$ \\
\hline & Grade 2 & 4 & $8 \%$ & 2 & $4 \%$ & 2 & $4 \%$ & 0 & $0 \%$ \\
\hline & Grade 3 & 4 & $8 \%$ & 0 & $0 \%$ & 0 & $0 \%$ & 0 & $0 \%$ \\
\hline & Grade 4 & 0 & $0 \%$ & 0 & $0 \%$ & 0 & $0 \%$ & 0 & $0 \%$ \\
\hline & Overall & 11 & $22 \%$ & 29 & $58 \%$ & 4 & $8 \%$ & 0 & $0 \%$ \\
\hline \multirow{5}{*}{ Cycle 2} & Grade 1 & 14 & $28 \%$ & 27 & $54 \%$ & 3 & $6 \%$ & 0 & $0 \%$ \\
\hline & Grade 2 & 4 & $8 \%$ & 3 & $6 \%$ & 2 & $4 \%$ & 0 & $0 \%$ \\
\hline & Grade 3 & 3 & $6 \%$ & 0 & $0 \%$ & 0 & $0 \%$ & 2 & $4 \%$ \\
\hline & Grade 4 & 4 & $8 \%$ & 0 & $0 \%$ & 0 & $0 \%$ & 2 & $4 \%$ \\
\hline & Overall & 25 & $50 \%$ & 30 & $60 \%$ & 5 & $10 \%$ & 4 & $8 \%$ \\
\hline \multirow{6}{*}{ Cycle 3} & Grade 1 & 17 & $34 \%$ & 19 & $38 \%$ & 3 & $6 \%$ & 0 & $0 \%$ \\
\hline & Grade 2 & 3 & $6 \%$ & 12 & $24 \%$ & 3 & $6 \%$ & 1 & $2 \%$ \\
\hline & Grade 3 & 0 & $0 \%$ & 2 & $4 \%$ & 4 & $8 \%$ & 2 & $4 \%$ \\
\hline & Grade 4 & 8 & $16 \%$ & 0 & $0 \%$ & 0 & $0 \%$ & 0 & $0 \%$ \\
\hline & Grade 5 & 0 & $0 \%$ & 0 & $0 \%$ & 0 & $0 \%$ & 2 & $4 \%$ \\
\hline & Overall & 28 & $56 \%$ & 33 & $66 \%$ & 10 & $20 \%$ & 5 & $10 \%$ \\
\hline
\end{tabular}

frequently encountered toxicity was anemia $(n=29,58 \%)$ which was mainly grade 1 or 2 . Grade $3 / 4$ neutropenia $(n=11,22 \%)$ was seen in $8 \%$. Thrombocytopenia was $(\mathrm{n}=4,8 \%)$ mainly grade 1 or 2 . Among Gastrointestinal toxicities (Table 3), nausea was most common $(\mathrm{n}=31,62 \%)$. Diarrhea was the second most common toxicity $(n=18,38 \%)$ with grade 2 diarrhea observed in $28 \%$, grade 
1 in $6 \%$ and grade 3 in $4 \%$ of patients. Grade 2 abdominal pain was seen in $18 \%$, grade 1 in $6 \%$ and grade 3 in $4 \%$ of patients. Vomiting was seen in $22 \%$, grade 1 in $12 \%$ and grade 2 in $10 \%$ of patients. Constipation and mucositis were seen in $14 \%$ of patients which were all grade 1 . Among the other toxicities reported (Table 4$)$ in cycle 1 , fatigue was most common $(n=33,66 \%)$ with grade 1 toxicity

Table 3. Chemotherapy associated gastrointestinal toxicities observed in our study group.

\begin{tabular}{|c|c|c|c|c|c|c|c|c|c|c|c|c|c|}
\hline \multirow{3}{*}{ Chemotherapy } & \multirow{3}{*}{ Toxicity grade } & \multicolumn{12}{|c|}{ Gastro-intestinal toxicities } \\
\hline & & \multicolumn{2}{|c|}{ Nausea } & \multicolumn{2}{|c|}{ Vomiting } & \multicolumn{2}{|c|}{ Diarrhoea } & \multicolumn{2}{|c|}{ Constipation } & \multicolumn{2}{|c|}{ Oral mucositis } & \multicolumn{2}{|c|}{ Abdominal pain } \\
\hline & & $\mathrm{n}$ & $\%$ & $\mathbf{n}$ & $\%$ & $\mathrm{n}$ & $\%$ & $\mathrm{n}$ & $\%$ & $\mathrm{n}$ & $\%$ & $\mathrm{n}$ & $\%$ \\
\hline \multirow{5}{*}{ cycle 1} & Grade 1 & 29 & $58 \%$ & 6 & $12 \%$ & 3 & $6 \%$ & 7 & $14 \%$ & 7 & $14 \%$ & 3 & $6 \%$ \\
\hline & Grade 2 & 2 & $4 \%$ & 5 & $10 \%$ & 14 & $28 \%$ & 0 & $0 \%$ & 0 & $0 \%$ & 9 & $18 \%$ \\
\hline & Grade 3 & 0 & $0 \%$ & 0 & $0 \%$ & 2 & $4 \%$ & 0 & $0 \%$ & 0 & $0 \%$ & 2 & $4 \%$ \\
\hline & Grade 4 & 0 & $0 \%$ & 0 & $0 \%$ & 0 & $0 \%$ & 0 & $0 \%$ & 0 & $0 \%$ & 0 & $0 \%$ \\
\hline & Overall & 31 & $62 \%$ & 11 & $22 \%$ & 19 & $38 \%$ & 7 & $14 \%$ & 7 & $14 \%$ & 14 & $28 \%$ \\
\hline \multirow{5}{*}{ cycle 2} & Grade 1 & 34 & $68 \%$ & 8 & $16 \%$ & 4 & $8 \%$ & 3 & $6 \%$ & 4 & $8 \%$ & 6 & $12 \%$ \\
\hline & Grade 2 & 4 & $8 \%$ & 12 & $24 \%$ & 8 & $16 \%$ & 0 & $0 \%$ & 2 & $4 \%$ & 7 & $14 \%$ \\
\hline & Grade 3 & 0 & $0 \%$ & 2 & $4 \%$ & 5 & $10 \%$ & 0 & $0 \%$ & 0 & $0 \%$ & 0 & $0 \%$ \\
\hline & Grade 4 & 0 & $0 \%$ & 0 & $0 \%$ & 0 & $0 \%$ & 0 & $0 \%$ & 0 & $0 \%$ & 0 & $0 \%$ \\
\hline & Overall & 38 & $76 \%$ & 22 & $44 \%$ & 17 & $34 \%$ & 3 & $6 \%$ & 9 & $18 \%$ & 13 & $26 \%$ \\
\hline \multirow{6}{*}{ cycle 3} & Grade 1 & 29 & $58 \%$ & 2 & $4 \%$ & 3 & $6 \%$ & 0 & $0 \%$ & 4 & $8 \%$ & 3 & $6 \%$ \\
\hline & Grade 2 & 6 & $12 \%$ & 10 & $20 \%$ & 9 & $18 \%$ & 0 & $0 \%$ & 0 & $0 \%$ & 10 & $20 \%$ \\
\hline & Grade 3 & 0 & $0 \%$ & 2 & $4 \%$ & 3 & $6 \%$ & 0 & $0 \%$ & 2 & $4 \%$ & 2 & $4 \%$ \\
\hline & Grade 4 & 0 & $0 \%$ & 0 & $0 \%$ & 0 & $0 \%$ & 0 & $0 \%$ & 0 & $0 \%$ & 0 & $0 \%$ \\
\hline & Grade 5 & 0 & $0 \%$ & 0 & $0 \%$ & 0 & $0 \%$ & 0 & $0 \%$ & 0 & $0 \%$ & 0 & $0 \%$ \\
\hline & Overall & 35 & $70 \%$ & 14 & $28 \%$ & 15 & $30 \%$ & 0 & $0 \%$ & 6 & $12 \%$ & 15 & $30 \%$ \\
\hline
\end{tabular}

Table 4. Chemotherapy associated other toxicities observed in our study group.

\begin{tabular}{|c|c|c|c|c|c|c|c|c|c|c|c|c|c|}
\hline \multirow{3}{*}{ Chemotherapy } & \multirow{3}{*}{ Toxicity Grade } & \multicolumn{12}{|c|}{ Other toxicities } \\
\hline & & \multicolumn{2}{|c|}{ Fatigue } & \multicolumn{2}{|c|}{$\begin{array}{l}\text { Peripheral } \\
\text { neuropathy }\end{array}$} & \multicolumn{2}{|c|}{ Alopecia } & \multicolumn{2}{|c|}{$\begin{array}{c}\text { Sr. Bilirubin } \\
\text { elevation }\end{array}$} & \multicolumn{2}{|c|}{$\begin{array}{l}\text { AST/ALT elevation } \\
\text { elevation }\end{array}$} & \multicolumn{2}{|c|}{ Pedal edema } \\
\hline & & $\mathrm{n}$ & $\%$ & $\mathrm{n}$ & $\%$ & $\mathrm{n}$ & $\%$ & $\mathrm{n}$ & $\%$ & $\mathrm{n}$ & $\%$ & $\mathrm{n}$ & $\%$ \\
\hline \multirow{4}{*}{ Cycle 1} & Grade 1 & 27 & $54 \%$ & 8 & $16 \%$ & 11 & $22 \%$ & 0 & $0 \%$ & 0 & $0 \%$ & 0 & $0 \%$ \\
\hline & Grade 2 & 6 & $12 \%$ & 0 & $0 \%$ & 2 & $4 \%$ & 0 & $0 \%$ & 0 & $0 \%$ & 0 & $0 \%$ \\
\hline & Overall & 33 & $66 \%$ & 8 & $16 \%$ & 13 & $26 \%$ & 0 & $0 \%$ & 0 & $0 \%$ & 0 & $0 \%$ \\
\hline & Grade 1 & 37 & $74 \%$ & 15 & $30 \%$ & 23 & $46 \%$ & 1 & $2 \%$ & 1 & $2 \%$ & 4 & $8 \%$ \\
\hline \multirow[t]{3}{*}{ Cycle 2} & Grade 2 & 6 & $12 \%$ & 2 & $4 \%$ & 13 & $26 \%$ & 0 & $0 \%$ & 0 & $0 \%$ & 0 & $0 \%$ \\
\hline & Overall & 43 & $86 \%$ & 17 & $34 \%$ & 36 & $72 \%$ & 1 & $2 \%$ & 1 & $2 \%$ & 4 & $8 \%$ \\
\hline & Grade 1 & 33 & $66 \%$ & 14 & $28 \%$ & 7 & $14 \%$ & 0 & $0 \%$ & 0 & $0 \%$ & 6 & $12 \%$ \\
\hline \multirow[t]{2}{*}{ Cycle 3} & Grade 2 & 12 & $24 \%$ & 6 & $12 \%$ & 31 & $62 \%$ & 0 & $0 \%$ & 0 & $0 \%$ & 2 & $4 \%$ \\
\hline & Overall & 45 & $90 \%$ & 20 & $40 \%$ & 38 & $76 \%$ & 0 & $0 \%$ & 3 & $6 \%$ & 8 & $16 \%$ \\
\hline
\end{tabular}


seen in $54 \%$ and grade 2 in $12 \%$. Other reported toxicities were peripheral neuropathy $(\mathrm{n}=8,16 \%)$ and alopecia seen in $26 \%$ [grade $1(22 \%)$, grade $2(4 \%)$ ].

Post cycle 2, anemia was again the most frequently detected toxicity $(\mathrm{n}=30$, $60 \%)$ which was predominantly grade $1(\mathrm{n}=27,54 \%)$. Neutropenia was seen in $50 \%$ [grade 1 (28\%), grade $2(8 \%)$, grade $3(6 \%)$, and grade $4(8 \%)$ ]. Febrile neutropenia was seen in $8 \%$. Thrombocytopenia was seen in $10 \%$ [grade $1(6 \%)$ and grade $2(4 \%)$ ] (Table 2). The most frequent GI toxicity was nausea $(\mathrm{n}=38,76 \%)$ and vomiting [44\%; grade $1(16 \%)$, grade $2(24 \%)$, and grade $3(4 \%)$ ]. Diarrhea was seen in $34 \%$ of patients [grade $1(8 \%)$, grade $2(16 \%)$, and grade $3(10 \%)$ ]. Abdominal pain was seen in $26 \%$ of patients [grade $1(12 \%)$ and grade $2(14 \%)$ ] and oral mucositis in $18 \%$ [grade 1 (8\%) and grade $2(4 \%)$ ] (Table 3). Among other common toxicities, fatigue was most common seen in $86 \%$ [grade 1 (72\%) and grade $2(12 \%)$ ]. Alopecia was the second most common toxicity seen in $72 \%$ [grade $1(46 \%)$ and grade $2(26 \%)$ ]. Neuropathy was seen in $34 \%$ which was grade 1 in $30 \%$ and grade 2 in $4 \%$ of patients. Pedal edema was seen in $8 \%$ of patients, all of which were grade 1 (Table 4 ).

The most common hematological toxicity in $3^{\text {rd }}$ cycle was anemia seen in $66 \%$ of patients [grade $1(38 \%)$, grade $2(24 \%)$ and grade $3(4 \%)$ ]. Febrile neutropenia was seen in $10 \%$ [grade $2(2 \%)$, grade $3(4 \%)$ and grade $5(4 \%)$ ] which was fatal in 2 patients (Table 2). In $3^{\text {rd }}$ cycle, nausea was the most common gastrointestinal toxicity seen in $70 \%$ which was mainly grade 1 [58\%] and grade 2 [12\%]. Abdominal pain was seen in 30\% [grade $2(20 \%)$, grade $1(6 \%)$ and grade $3(4 \%)$ ], diarrhea in $30 \%$ [grade $2(18 \%)$, grade $3(6 \%)$ and grade $1(6 \%)$ ] and vomiting was seen in $28 \%$ of patients [grade $2(20 \%)$, grade 1 and grade $3(4 \%)$ ]. Oral mucositis was seen in $12 \%$ of patients [grade $1(8 \%)$ and grade $3(4 \%)$ ] (Table 3 ).

Neuropathy was seen in $40 \%$ [grade $1(28 \%)$ and grade $2(12 \%)$ ]. The other common toxicities are depicted in (Table 4).

Dose modifications were required only in $14(28 \%)$ patients. These modifications were done for Grade 3/4 toxicity.

\subsubsection{Efficacy Responses as Assessed by PET-CT Scan}

\section{1) ORR [Overall Response Rate] and DCR [Disease Control Rate]}

In this study we observed an ORR [CR + PR] of $68 \%$ and DCR [CR + PR + $\mathrm{SD}$ ] of $96 \%$ which indicated the effectiveness of DOF regimen (Figure 1).

\section{2) Overall Survival (OS) Rate at One Year}

The overall survival rate at one year was $88 \%$ (Figure 2).

\subsubsection{Resectability Rate}

40 [80\%] patients were able to undergo surgical resection which indicated the effectiveness and good tolerance to chemotherapy.

1) Pathological CR (pCR) and Node negativity post neoadjuvant chemotherapy $\left[y p N_{0}\right]$ status

Pathological CR indicates no disease after surgical resection post neo-adjuvant treatment and it was seen in 7 [17.5\%] patients in our study. $y p N_{0}$ which indi- 
cates no pathological involved lymph nodes after neo-adjuvant therapy was found to be $42.5 \%$ (17 patients). pCR was a good predictor of survival.

2) Comparison of mean Disease free survival (DFS) and pathological CR [pCR]

The mean DFS [in months] in patients with or without pCR was $11.46 \pm 1.35$ and $8.53 \pm 2.21$ respectively $[\mathrm{P}=0.002]$. This indicated that patients achieving pCR had better DFS than patients who did not achieve pCR (Table 5).

3) Comparison of mean DFS and $y p N_{0}$ status

The mean DFS [in months] in patients with or without $y p N_{0}$ status was 10.27

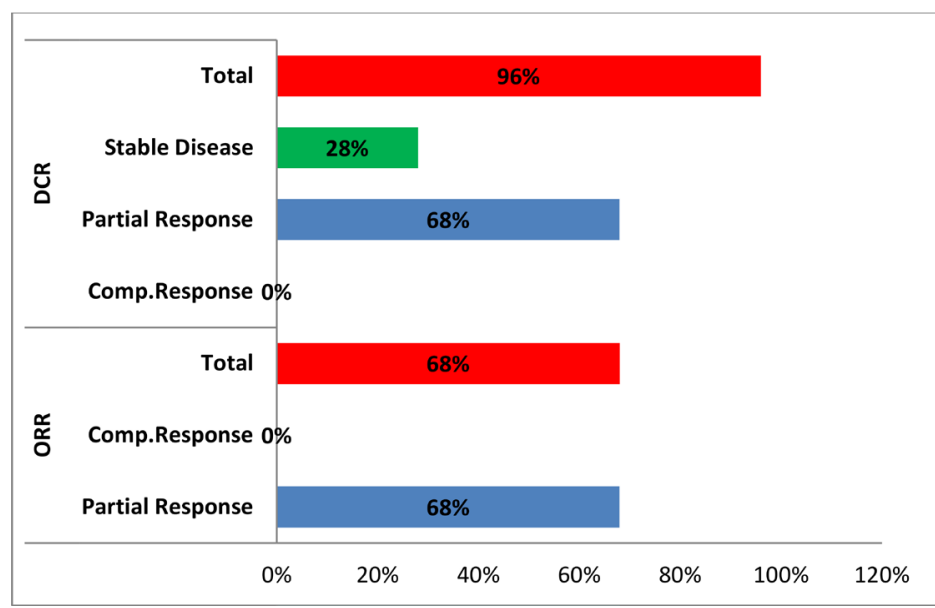

Figure 1. Assessment of ORR [Overall response rate] and DCR [Disease control rate] by CT scan.

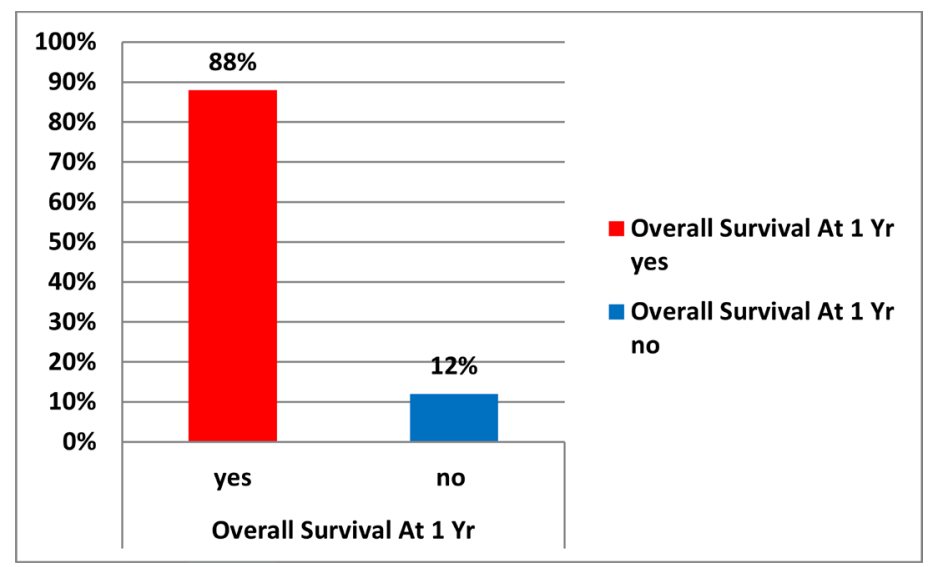

Figure 2. Overall survival (OS) rate at one year.

Table 5. Comparison of mean DFS and pathological CR [pCR].

\begin{tabular}{cccccccc}
\hline \multicolumn{7}{c}{ Comparison of mean DFS based on pathological CR condition in study patients using } \\
Independent Student $t$ test \\
\hline Variables & Pathological CR & N & Mean & SD & Mean Diff & t & P-Value \\
\hline \multirow{2}{*}{ DFS } & Yes & 7 & 11.46 & 1.35 & & & \\
& No & 33 & 8.53 & 2.21 & & & \\
\hline
\end{tabular}


\pm 1.92 and $8.14 \pm 2.27$ respectively $[\mathrm{P}=0.003]$. Patients who achieved $y p N_{0}$ status on surgical resection post neo-adjuvant chemotherapy had better DFS than patients who didn't (Table 6).

4) Comparison of mean DFS and pathological tumor [T] down staging and pathological nodal [N] down staging

Tumor [T] down staging was achieved in 26 patients. The mean DFS [in months] in patients having [T] down staging was $9.49 \pm 2.47$ compared to $8.21 \pm$ 1.95 in those who did not $[\mathrm{P}=0.10]$. There was no statistically significant survival benefit.

Similarly, pathological nodal down staging was assessed and nodal [N] down staging was achieved in 26 patients. The mean DFS [in months] in patients having $[\mathrm{N}]$ down staging was $10.02 \pm 1.78$ compared to $7.23 \pm 2.26$ in those who did not $[\mathrm{P}<0.001]$ (Table 7).

\section{Discussion}

Neoadjuvant chemotherapy (NACT) is now used worldwide as initial therapy for treating LAGC and operable gastric cancer. It has shown improvement in survival when compared to surgery alone [10] [11]. In our study we evaluated the efficacy and safety profile of patients who received neo-adjuvant chemotherapy with DOF regimen. We broadly categorized the toxicities into hematological, gastrointestinal and others.

The most common hematological toxicities observed in our study included

Table 6. Comparison of mean DFS and $y p N_{0}$ status.

Comparison of mean DFS based on pathological and non-pathological $y p N_{0}$ in study patients using Independent Student $t$ test

\begin{tabular}{cccccccc}
\hline Variables & $y p N_{0}$ Status & N & Mean & SD & Mean Diff & t & P-Value \\
\hline \multirow{2}{*}{ DFS } & $y p N_{0}$ & 17 & 10.27 & 1.92 & & & \\
& Non- $y p N_{0}$ & 23 & 8.14 & 2.27 & & & \\
& & &
\end{tabular}

Table 7. Comparison of mean DFS and Tumor down staging [T] and Nodal down staging $[\mathrm{N}]$.

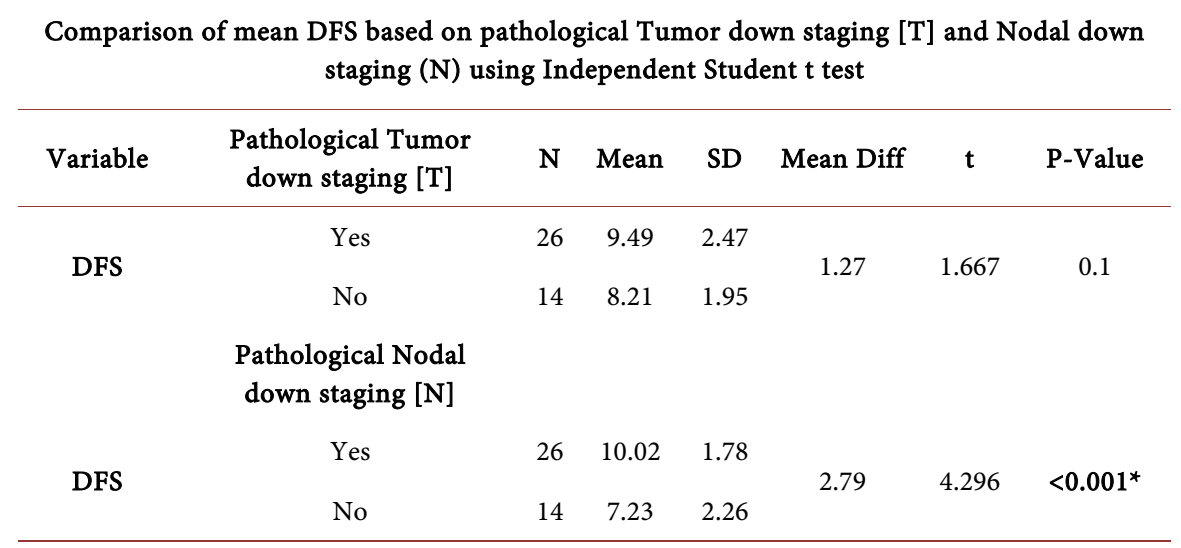


anemia (cycle 1: $58 \%$ cycle 2: $60 \%$; cycle 3: $66 \%$ Overall: $61.2 \%$ ), neutropenia (cycle 1: $22 \%$ cycle 2: $50 \%$; cycle 3: $56 \%$ Overall $42.6 \%$,), thrombocytopenia (cycle 1: $8 \%$, cycle 2: $10 \%$; cycle 3: $20 \%$ Overall: $13.2 \%$ ) and febrile neutropenia (cycle 1: $0 \%$, cycle 2: $8 \%$, cycle 3: $10 \%$ Overall: $6 \%$ ). The hematological toxicity profile of DOF regimen reported by Liu M et al. included anemia (50\%), neutropenia (44.8\%), thrombocytopenia (36.2\%) and febrile neutropenia (10.3\%). This was comparable to our study except a lower incidence of thrombocytopenia and febrile neutropenia [12]. The slightly higher percentage of anemia in our study can be partly attributed to lower baseline hemoglobin levels [13]. The hematological toxicity profile of DOF regimen reported by Yao $\mathrm{Z}$ et al. included anemia (80\%), neutropenia (84\%), thrombocytopenia (22\%) and febrile neutropenia (18\%) [8]. The lower percentage of anemia, neutropenia and febrile neutropenia in our study could be due to lower doses of Docetaxel and Oxaliplatin used in our study. The most common Gastro-Intestinal [GI] toxicities observed in our study included nausea (cycle 1: $62 \%$ cycle 2: $76 \%$; cycle 3: $70 \%$ Overall: $69.2 \%$ ), vomiting (cycle 1: $22 \%$ cycle $2: 44 \%$; cycle $3: 28 \%$ Overall $31.2 \%$ ), diarrhea (cycle 1: $38 \%$ cycle 2: $34 \%$; cycle 3: $30 \%$ Overall: $34 \%$ ), abdominal pain (cycle 1: $28 \%$, cycle 2: $26 \%$, cycle $3: 30 \%$ Overall: $28 \%$ ) and oral mucositis (cycle 1: $14 \%$, cycle 2: $18 \%$ cycle 3: $12 \%$ Overall: $14 \%$ ). A study done by Van Cutsem E et al. showed GI toxicities as follows: nausea (59\%), vomiting (35\%), diarrhea (67\%), abdominal pain (22\%), and oral mucositis (33\%) [14]. These findings suggest that our study had lesser incidence of diarrhea, oral mucositis and constipation.

There was a steady increase in the number of patients who developed neutropenia and anemia with each cycle of DOF. This could be attributed to dose related cumulative toxicities of the component drugs which gradually increase with each cycle. Unlike hematological toxicities, the gastrointestinal adverse effects did not show an increasing trend with each cycle. This may be due to better GI tolerability of the DOF regimen in Indian patients. GI tolerability is one of the important factors which influence patient compliance to chemotherapeutic regimens and thus influence the effectiveness of therapy [14] [15].

The other common toxicities observed in our study included fatigue (cycle 1: 66\% cycle 2: $86 \%$; cycle 3: $90 \%$ Overall: $80.6 \%$ ), alopecia (cycle 1: $26 \%$ cycle 2 : 72\%; cycle 3: 76\% Overall 58\%) and peripheral neuropathy (cycle 1: $16 \%$, cycle 2 : $34 \%$, cycle 3: 40\% Overall: $30 \%$ ). Retrospective studies show a slightly higher incidence of fatigue, peripheral neuropathy and alopecia in docetaxel based regimens [16]. In a study by Yao $\mathrm{Z}$ et al., the frequency of alopecia and peripheral neuropathy was $56 \%$ and $58 \%$ respectively. The lower incidence of neuropathy in our study can be explained by modified doses of oxaliplatin, lesser number of patients with diabetes and lesser number of chemotherapy cycles used [17]. These adverse effects were managed and did not require dose modifications.

PR was achieved in $68 \%$ and SD in $28 \%$ of patients. ORR [CR + PR] achieved in our study was $68 \%$ which is similar to the ORR achieved in a study by Wang $\mathrm{Z}$ et al. at 66.6\% [18]. The ORR achieved in our study was higher than most oth- 
er studies namely Yao $\mathrm{Z}$ et al. and Liu M et al. which showed ORR of $42.2 \%$ and $50 \%$ respectively [8] [12]. Our study had increased Response Rates [ORR and DCR] as compared to other studies. This could be attributed to higher number of patients with good Performance Status in our study [ECOG $1=82 \%$ ] when compared to study by Yao Z et al. [ECOG $=60 \%$ ], more number of patients $<60$ years [60\%] resulting in better tolerability of chemotherapy which is reflected by lesser dose modifications [28\%] when compared to Yao Z et al. [64\%, [8] [19]].

The DFS and PFS [Disease Free Survival and Progression free survival] in our study were 9.1 months and 14.2 months respectively which is higher compared to other studies. A study done by Satheesh et al. reported a median PFS of 9 months and a similar study done by Liu M reported a median PFS 8.2 months [12] [20]. The patients in our study were entirely LAGC where as other studies included both LAGC and metastatic patients. It is well known that patients with good performance status at baseline [ECOG 0 and 1] have better survival [19]. In our study most of the patients had an ECOG performance status of 1 [82\%].

In our study the pathological CR [pCR] rates was $17.5 \%$ which is similar to studies by Al Batran et al. who reported a pCR of $18 \%$ [7]. Our study found a statistically significant difference in DFS in those who achieved pCR (11.46 months) compared to those who did not (8.53 months), $\mathrm{P}<0.05$. Several other studies have shown similar positive co-relation between pCR and survival [21] [22] and [23].

Several studies achieving $y p N_{0}$ disease status showed an improved survival when compared to residual lymph node disease. In a study done by Ikoma $\mathrm{N}$ et al., 59\% of patients achieved $y p N_{0}$ status and these patients had better survival than those with $y p N+$ status [24] [25] [26]. In our study $y p N_{0}$ status was achieved in $42.5 \%$ of patients and they had better DFS [10.27 months] compared to those who did not [8.14 months] which was statistically significant ( $\mathrm{P}$ value $<0.005)$.

Studies have shown that patients achieving pathological nodal down staging have better DFS compared to those who did not achieve nodal down staging, whereas pathological tumor down staging does not appear to provide any survival benefit [26] [27]. In our study there was a statistically significant survival benefit [ $\mathrm{P}$ value $<0.05$ ] in patients achieving nodal down staging [10.02 months] as compared to those who did not [7.23 months]. Survival benefit was not statistically significant with respect to tumor down staging.

\section{Limitations of the Study}

Our study was mainly limited due to small sample size and a single arm design.

\section{Conclusion}

Our study shows that the DOF regimen can be an effective and feasible option as NACT in the management of patients with LAGC in an Indian population. The safety profile of DOF regimen was favorable with majority of patients tolerating the regimen well. The Overall Response Rates, Disease Control Rates and Resec- 
tability Rates were higher compared to western studies. Pathological CR, $y p N_{0}$ status and nodal down staging, all showed positive correlation with survival outcomes. Further evaluation of DOF regimen with multi-centric studies involving large population in Indian settings would be needed to validate the outcomes and thus aid in effective management of patients with LAGC.

\section{Ethical Approval}

Ethical approval was obtained from the institution ethics committee.

\section{Conflicts of Interest}

The authors declare no conflicts of interest regarding the publication of this paper.

\section{References}

[1] Ajani, J.A., et al. (2016) Gastric Cancer, Version 3.2016, NCCN Clinical Practice Guidelines in Oncology. Journal of the National Comprehensive Cancer Network, 14, 10. https://jnccn.org/view/journals/jnccn/14/10/article-p1286.xml

[2] Smyth, E.C., et al. (2016) Gastric Cancer: ESMO Clinical Practice Guidelines. Annals of Oncology, 27, v38-v49.

https://www.esmo.org/guidelines/gastrointestinal-cancers/gastric-cancer https://doi.org/10.1093/annonc/mdw350

[3] Ibrahim, M. and Gilbert, K. (2017) Management of Gastric Cancer in Indian Population. Translational Gastroenterology and Hepatology, 2, 64. https://doi.org/10.21037/tgh.2017.07.02

[4] Dikshit, R.P., Mathur, G., Mhatre, S. and Yeole, B.B. (2011) Epidemiological Review of Gastric Cancer in India. Indian Journal of Medical and Pediatric Oncology, 32, 3-11. https://doi.org/10.4103/0971-5851.81883

[5] Becker, K., Mueller, J.D., Schulmacher, C., Ott, K., et al. (2003) Histopathology and Grading of Regression in Gastric Carcinoma Treated with Neoadjuvant Chemotherapy. Cancer, 98, 1521-1530. https://doi.org/10.1002/cncr.11660

[6] De Manzoni, G., Marrelli, D., Baiocchi, G.L., Morgagni, P., et al. (2017) The Italian Research Group of Gastric Cancer (GIRCG) Guidelines for Gastric Cancer Staging and Treatment: 2015. Gastric Cancer, 20, 20-30. https://doi.org/10.1007/s10120-016-0615-3

[7] Al-Batran, S.E., Homann, N., Pauligk, C., Goetze, T.O., Meiler, J., Kopp, H.G., et al. (2017) Perioperative Chemotherapy with Docetaxel, Oxaliplatin, and Fluorouracil/Leucovorin (FLOT) versus Epirubicin, Cisplatin, and Fluorouracil or Capecitabine (ECF/ECX) for Resectable Gastric or Gastroesophageal Junction (GEJ) Adenocarcinoma (FLOT4-AIO): A Multicenter, Randomized Phase 3 Trial. Journal of Clinical Oncology, 35, 4004-4005.

[8] Yao, Z., Guo, H., Yuan, Y., et al. (2014) Retrospective Analysis of Docetaxel, Oxaliplatin plus Flurouracil Compared with Epirubicin, Cisplatin and Flurouracil as First Line Therapy for Advanced Gastric Cancer. Journal of Chemotherapy, 26, 117-121.

http://www.embase.com/search/results?subaction=viewrecord\&from=export\&id=L $\underline{372560129}$

https://doi.org/10.1179/1973947813Y.0000000120 
[9] Young, K., Minchom, A., Cunningham, D., Chau, I., et al. (2012) Neoadjuvant Chemotherapy for Gastric Cancer. Translational Gastrointestinal Cancer, 1, 202-204.

[10] Chen, W., Shen, J., Pan, T., et al. (2014) FOLFOX versus EOX as a Neoadjuvant Chemotherapy Regimen for Patients with Advanced Gastric Cancer. Experimental and Therapeutic Medicine, 7, 461-467. https://doi.org/10.3892/etm.2013.1449

[11] Iwasaki, Y., Sasako, M., Yamamoto, S., Nakamura, K., et al. (2013) Phase II Study of Preoperative S-1 and Cisplatin in Patients with Clinically Resectable Type 4 and Large Type 3 Gastric Cancer. Japan Clinical Oncology Group Study (JCOG0210). Journal of Surgical Oncology, 107, 741-745. https://doi.org/10.1002/jso.23301

[12] Liu, M., Hu, G., Wang, Y., Guo, J., Liu, L., et al. (2018) Comparison of FOLFOX and DOF Regimens as First-Line Treatment in East Asian Patients with Advanced Gastric Cancer. Oncotargets and Therapy, 11, 375-381.

https://doi.org/10.2147/OTT.S149624

[13] Kumari, R., Bharti, R.K., Singh, K., et al. (2017) Prevalence of Iron Deficiency and Iron Deficiency Anemia in Adolescent Girls in a Tertiary Care Hospital. Journal of Clinical and Diagnostic Research, 11, 4-6. https://doi.org/10.7860/JCDR/2017/26163.10325

[14] Van Cutsem, E., Boni, C., Tabernero, J., Massuti, B., et al. (2015) Docetaxel plus Oxaliplatin with or without Fluorouracil or Capecitabine in Metastatic or Locally Recurrent Gastric Cancer: A Randomized Phase II Study. Annals of Oncology, 26, 149-156.

http://www.embase.com/search/results?subaction=viewrecord\&from=export\&id=L $\underline{602211800}$ https://doi.org/10.1093/annonc/mdu496

[15] Park, S.C. and Chun, H.J. (2013) Chemotherapy of Advanced Gastric Cancer: Review and Update of Current Practices. Gut and Liver, 7, 385-393.

https://doi.org/10.5009/gnl.2013.7.4.385

[16] Petrioli, R., Roviella, G., Zanotti, L., et al. (2016) Epirubicin-Based Compared with Docetaxel-Based Chemotherapy for Advanced Gastric Carcinoma: A Systematic Review and Meta-Analysis. Critical Reviews in Oncology/Hematology, 102, 82-88. https://doi.org/10.1016/j.critrevonc.2016.04.001

[17] Trivedi, M.S., Hershman, D.L., Crew, K.D., et al. (2015) Management of Chemotherapy-Induced Peripheral Neuropathy. The American Journal of Hematology/ Oncology, 11, 4-9.

[18] Wang, Z.H., Guo, J., Chen, Z. and Zhang, E.N. (2008) Preliminary Study of Biweekly Regimen of Docetaxel, Oxaliplatin, 5-Fluorouracil and Leucovorin for Advanced Gastric Cancer. Chinese Journal of Oncology, 30, 389-391.

[19] Shitara, K., Muro, K., Matsuo, K., et al. (2009) Chemotherapy for Patients with Advanced Gastric Cancer with Performance Status 2. Gastrointestinal Cancer: Research, 3, 220-224.

[20] Satheesh, T., Shashidhara, P., Ravi, T. and Shekar, P. (2017) Modified DOF Regimen in Advanced Stomach Cancer: A Single Center Experience from Bangalore. Annals of Oncology, 28, 44.

https://www.annalsofoncology.org/article/S0923-7534(19)65887-4/fulltext https://doi.org/10.1093/annonc/mdx261.104

[21] Li, Z., Shan, F., Wang, Y., et al. (2018) Correlation of Pathological Complete Response with Survival after Neoadjuvant Chemotherapy in Gastric or Gastro Esophageal Junction Cancer Treated with Radical Surgery: A Meta-Analysis. PLoS ONE, 13, 1-10. https://doi.org/10.1371/journal.pone.0189294 
[22] Fields, R.C., Strong, V.E., Gonen, M., et al. (2011) Recurrence and Survival after Pathologic Complete Response to Preoperative Therapy Followed by Surgery for Gastric or Gastro Esophageal Adenocarcinoma. British Journal of Cancer, 104, 1840-1847. https://doi.org/10.1038/bjc.2011.175

[23] Lorenzen, S., Thuss-Patience, P., Al-Batran, S.E., et al. (2013) Impact of Pathologic Complete Response on Disease-Free Survival in Patients with Esophagogastric Adenocarcinoma Receiving Preoperative Docetaxel-Based Chemotherapy. Annals of Oncology, 24, 2068-2073. https://doi.org/10.1093/annonc/mdt141

[24] Zanoni, A., Verlato, G., et al. (2016) ypN0: Does It Matter How You Get There? Nodal Downstaging in Esophageal Cancer. Annals of Surgical Oncology, 23, 998-1004. https://doi.org/10.1245/s10434-016-5440-8

[25] Ikoma, N., Estrella, J.S., Hofstetter, W., et al. (2018) Nodal Downstaging in Gastric Cancer Patients: Promising Survival If ypNO Is Achieved. Annals of Surgical Oncology, 25, 2012-2017. https://doi.org/10.1245/s10434-018-6471-0

[26] Smyth, E.C., Fassan, M., Cunningham, D., et al. (2016) Effect of Pathologic Tumor Response and Nodal Status on Survival in the Medical Research Council Adjuvant Gastric Infusional Chemotherapy Trial. Journal of Clinical Oncology, 34, 2721-2727. https://doi.org/10.1200/JCO.2015.65.7692

[27] Ronellenfitsch, U., Schwarzbach, M., Hofheinz, R., et al. (2017) Predictors of Overall and Recurrence-Free Survival after Neoadjuvant Chemotherapy for Gastroesophageal Adenocarcinoma: Pooled Analysis of Individual Patient Data (IPD) from Randomized Controlled Trials (RCTs). European Journal of Surgical Oncology, 43, 1550-1558. https://doi.org/10.1016/j.ejso.2017.05.005 